\title{
Mathematical Formulation and Analysis of the Optimal Launch Timing for Mobile Applications with Perceived Value and Network Effect
}

\author{
Wei Li, Shu-gang Ma, Yan-peng He, and Qiao-zhi An \\ The School of Business, Hebei University of Economics and Business, Shijiazhuang 050061, China \\ Correspondence should be addressed to Wei Li; weilee@heuet.edu.cn
}

Received 3 March 2017; Revised 23 July 2017; Accepted 17 September 2017; Published 6 November 2017

Academic Editor: Frederic Kratz

Copyright (C) 2017 Wei Li et al. This is an open access article distributed under the Creative Commons Attribution License, which permits unrestricted use, distribution, and reproduction in any medium, provided the original work is properly cited.

\begin{abstract}
Successive release is a common strategy adopted by mobile app providers, and determining the launch timing of new app versions presents an important challenge to these providers. Network effect and consumers' perceived value are significant factors that influence the decisions of providers. By focusing on a monopoly market, we develop an optimization model that incorporates the two factors to determine the optimal launch timing of new versions of mobile apps. The model is solved by Lagrangian method, and the closed-form results indicate that the monopoly provider launches new app versions as soon as possible if the consumers' perceived value is not sufficiently high. Otherwise, the new version is launched after (or before) the sales of its former version reach maturity if the network effect is (or not) sufficiently high. Moreover, the monopoly app vendor delays the launch of a new version when the consumers enjoy a large network externality; however, the same vendor accelerates the release of upgrades if the consumers have a high perceived value of the app. This paper presents a novel mathematical formulation to analyze the launching policy of digital products.
\end{abstract}

\section{Introduction}

Providers of mobile applications (commonly referred to as mobile "apps") often adopt the successive release strategy to attract more users and keep their competitive advantage. Most of the apps being used today represent improved versions of their earlier generations, and such products will be substituted over time by even newer generations. When the firms launch their new products into markets, timing is one of the important factors for their profits [1]. Therefore, in the presence of successive product generations, the timing of introducing a new generation is a critical decision for an astute app publisher (the current work focuses on the following three types of app: (1) the paid app; (2) the app with free trial version but paid premium services; (3) the app that permits user to download for free, but enables some functionalities through in-app purchase).

Mobile apps are digital products, and the market decisions of such products should be based on their value to the consumers [2]. In the academia, consumer value is deemed as a new source of competitive advantage [3]. A significant feature of consumer value of app is that the consumers' perceived value often differs from the true value of the app. Unlike physical goods, some digital products, such as software, movies, and music, have a peculiar property wherein a consumer may not be able to assess these goods accurately and reliably before their consumption $[4,5]$. Sometimes, the process of evaluating such digital goods per se is the very process of consumption. A consumer's perceived value can influence his/her willingness to adopt the product and is considered by many a source of market success or failure. As another important feature, an app's value is determined not only by its inherent value but also by the network effect of the products. When influenced by a positive network effect, the value of an app to each user increases along with the size of the installed base $[6,7]$. Therefore, if app providers take products' consumer value into account, then the consumers' perceived value and network effect are two key factors that impact the launch timing decision of the provider. 
The primary goal of this work is to present a theoretical model for exploring how the two factors, namely, consumers' perceived value and network effect, can influence the optimal market entry timing of an app. We develop an analytical model for deciding such timing and compare our findings with those reported in the literature. This model is based on the Bass model [8] and incorporates both the consumers' perceived value and network effect. The analytical results show that if the consumers' perceived value is relatively low, then the new versions should be launched as soon as possible; however, if the consumers' perceived value is sufficiently high, then a new version may be launched before or after the sales of its former version have reached maturity, depending on the app's network effect.

The rest of this paper is organized as follows. In Section 2, we review the existing work on the market entry timing of new products. In Section 3, we develop an analytical model to explore the problems in the launch timing of new app versions in consideration of the network effect and the consumers' perceived value. In Section 4 , we investigate the optimal new version release policy of the app publisher and explore the mechanism of consumers' perceived value and network effect on the app's release timing. In Section 5, we discuss the managerial implications of our model and possible directions for future research.

\section{Related Works}

Our study is closely related to the market entry strategy for new products, which is a long-standing research topic that has been studied in various streams of literature in new product development, marketing, and economics.

The previous studies on this topic can be broadly classified into two categories. The first category incorporates product value to determine the optimal entry timing of the next product generation. Moorthy and Png [9] check whether the new product is introduced simultaneously or sequentially with respect to its former generation. They argue that sequential introduction is better than simultaneous introduction when the new product can cannibalize its former generation and is less attractive to consumers than to sellers. Fishman and Rafael [10] analyze optimal intervals between product generations and find that accelerating the release of upgrades is beneficial to the profits and social welfare of the firm. Ramachandran and Krishnan [11] study the impact of design architecture on the launch timing of a new product generation. They find that compared with those with an integrated architecture, those products with a modular architecture are more valuable to consumers and have a fast rate of upgrade.

Wang and Hui [12] explore the incentives of a monopolistic firm to delay the launch of an improved version of durable goods. They find that the time inconsistency problem drives the monopolist to delay its introduction of a new version even though the technologies are already mature. Mehra et al. [13] capture the balance between the costs and gains of introducing an upgraded version and show that such balance can increase the upgrade intervals of a product throughout its lifecycle. Choudhary and Zhang [14] determine an optimal release time for the software vendor in the context of cloud computing and SaaS and find that SaaS firms should launch their product early even though their product is far from perfect. Ebina et al. [15] analyze the entry timing decision of two firms using the spatial competition model and demonstrate that the incumbent has an incentive to locate closer to the center to delay the competitor's entry. Prasad et al. [16] focus on the movie industry and analyze the effect of customer expectations on the release timing of the video version that follows the theater version.

The second category considers the diffusion dynamics of existing products. Wilson and Norton [17] consider the entry timing for a product line extension with the assumption that this extension has a lower profit margin than the current product. They suggest a "now or never" policy in which the line extension is best introduced as early as possible or not be introduced at all. Mahajan and Muller [18] relax Wilson and Norton's assumption of decreased profit margins for the new product and claim that the introduction of a new product becomes optimal as soon as this product becomes available or when the sales of its previous version near its peak (they call this policy "now or at maturity"). Jiang et al. [19] find that the optimal entry timing of the successive version can also lie between now and maturity. Joshi et al. [20] find that new products should be introduced to the market either before or after the existing product reaches its peak sales depending on the leverage and backlash effect of the existing product. Krankel et al. [21] suggest that new product generations should be introduced when the technology of the existing generations is below a statedependent threshold. However, previous studies on product value do not consider diffusion dynamics, while those studies that incorporate diffusion dynamics do not consider product value. This research comprehensively considers both factors in determining the optimal launch timing of new product generations.

\section{Model Settings}

3.1. Products and Launch Timing. We present an analytical model with a monopolistic app publisher denoted as $M$. The monopolist $M$ develops and introduces the initial version $V_{0}$ at time $t_{0}$ and then launches $V_{1}$ at time $t_{1}>t_{0} . V_{1}$ is an improved version of $V_{0}$ and is compatible with $V_{0}$. The relationship between $V_{0}$ and $V_{1}$ is similar to PayPal v5 and PayPal v6 where the latter is the upgraded version of the former and the data generated in the former can be read or written in the latter. Following Krankel et al. [21] and Mehra et al. [13], who show that firms innovate based on the available technological frontier, we assume that $V_{i}(i=0,1)$ is the state of the art. This assumption is rational because, in a perfect world, mobile app publishers will leverage all possible opportunities to provide better utility to their customers.

3.2. Consumers' Utility. Given that $V_{i}$ is the state of the art, we assume that all consumers share the most frontier technologies. Therefore, these consumers obtain the same inherent value from $V_{i}$. We define the inherent value of $V_{i}$ as $q$. Mobile apps are intangible artifacts, and consumers are often unaware of the whole features of these apps. Therefore, 
the consumers' perceived value usually differs from the apps' true value. Inspired by Cheng and Liu [4], we define the consumers' perceived value as $\delta q$, where a high value of the coefficient $\delta$ indicates that the consumers highly evaluate the app.

Consumers' utility is also influenced by the network effect. According to [7], the network effect is determined by two factors, namely, its intensity and the user-installed base. We define the intensity of network effect as $\lambda$ and use the Bass model to express the user-installed base as shown in (1). $f(t)$ denotes the noncumulative number of adopters by time $t, m$ denotes the potential adopters, and $a$ and $b$ denote the coefficient of innovation and imitation, respectively. Therefore, those consumers who adopt the app at time $t$ will enjoy the network effect $\lambda \int_{t_{0}}^{t} f(t) d t$. The consumers' instantaneous utility by time $t$ is obtained in (2).

$$
\begin{aligned}
& f(t)=\frac{m(a+b)^{2}}{a} \frac{e^{-(a+b) t}}{\left[(b / a) e^{-(a+b) t}+1\right]^{2}} \\
& U(t)=\delta q+\lambda \int_{t_{0}}^{t} f(t) d t .
\end{aligned}
$$

3.3. Firm's Profit. The monopolist $M$ 's profit derives from the price that consumers pay for the app. Given its monopoly position in the market, $M$ can assign the price of an app (denoted as $P(t)$ ) to match the customers' utility as defined in (3) and to extract their full surplus. What needs to be stressed is that the price $P(t)$ is a general concept that refers to the fees that the consumers pay for the app. Specifically, $P(t)$ is at least one of the following three items: (1) the price that the consumers pay for a new version of an app; (2) the price that the consumers pay for an app's premium services of its trial version; (3) the price that the consumers pay for the inapp purchase that unlock additional functionalities. In fact, an app with premium services or available through in-app purchase could be regarded as the compatible upgrading of its initial version.

Therefore, the instantaneous revenue of $M$ from $V_{1}$ by time $t$ is denoted by $P(t) f(t)$. The cumulative revenue of $M$ from $V_{1}$ is the integral of instantaneous revenue. As its decision problem, the monopolist would determine the launch timing $t_{1}$ to maximize its cumulative profit from $V_{1}$. The optimization model is derived using (4) and (5), where the parameters $\pi$ and $T$ refer to the cumulative profit and planning horizon of $M$. Following Jiang et al. [19], the development cost of the app is not considered in our analysis, for the development cost of an app is the sunk cost that often exerts no impact on decision making.

$$
\begin{array}{ll}
P(t)= & U(t) \\
\max _{t_{1}} & \pi=\int_{t_{1}}^{T}\left[\delta q+\lambda \int_{t_{0}}^{t_{1}} f(t) d t\right] f(t) d t \\
\text { s.t. } & t_{0} \leq t_{1} \leq T .
\end{array}
$$

The Notations section summarizes the notations used in this work.

\section{Results and Analysis}

We solve the optimization model using the Lagrangian method, which has been widely used in previous studies such as [22]. The Lagrangian function of (4) is

$$
\begin{aligned}
L\left(t_{1}\right)= & \int_{t_{1}}^{T}\left[\delta q+\lambda \int_{t_{0}}^{t_{1}} f(t) d t\right] f(t) d t+\varepsilon\left(t_{1}-t_{0}\right) \\
& +\eta\left(T-t_{1}\right) .
\end{aligned}
$$

Given that the cumulative adopter at time $t$ (denoted as $F(t))$ is equal to the integration of $f(t)$, we obtain $F(t)=$ $\int f(t) d t=m\left(1-e^{-(a+b) t}\right) /\left((b / a) e^{-(a+b) t}+1\right)$. Following [19], we assume that the initial product, $V_{0}$, is introduced at time 0 , that is, $t_{0}=0$, and that the Lagrangian function (6) is equivalent to

$$
\begin{aligned}
L\left(t_{1}\right)= & {\left[\delta q+\lambda F\left(t_{1}\right)\right]\left[F(T)-F\left(t_{1}\right)\right]+\varepsilon t_{1} } \\
& +\eta\left(T-t_{1}\right) .
\end{aligned}
$$

The Kuhn-Tucker conditions require

$$
\begin{aligned}
\frac{\partial L}{\partial t_{1}} & =f\left(t_{1}\right)\left[\lambda F(T)-2 \lambda F\left(t_{1}\right)-\delta q\right]+\varepsilon-\eta \\
& =0 \\
\frac{\partial L}{\partial \varepsilon} & =t_{1} \geq 0, \\
\varepsilon\left(t_{1}-t_{0}\right) & =0 \\
\frac{\partial L}{\partial \eta} & =T-t_{1} \geq 0, \\
\eta\left(T-t_{1}\right) & =0
\end{aligned}
$$

Therefore, we obtain $\varepsilon=0$ and $\eta=0$ and then obtain the first-order condition as follows.

$$
\frac{\partial L}{\partial t_{1}}=f\left(t_{1}\right)\left[\lambda F(T)-2 \lambda F\left(t_{1}\right)-\delta q\right]=0 .
$$

We then obtain the second-order condition of (6), $\partial^{2} L / \partial t_{1}^{2}$, as follows.

$$
\frac{\partial^{2} L}{\partial t_{1}^{2}}=f^{\prime}\left(t_{1}\right)\left\{\lambda\left[F(T)-2 F\left(t_{1}\right)\right]-\delta q\right\}-2 \lambda f^{2}\left(t_{1}\right) .
$$

4.1. The Value of $\delta$ Is Not Sufficiently High. When the value of the parameter $\delta$ is not sufficiently high, technically, $\delta<$ $(1 / q)\left\{\lambda\left[\int_{t_{0}}^{T} f(t) d t-2 \int_{t_{0}}^{t_{1}} f(t) d t\right]-2 \lambda f^{2}\left(t_{1}\right) / f^{\prime}\left(t_{1}\right)\right\}$, equation (11) is established, and we can derive that $\partial^{2} L / \partial t_{1}^{2}>0$. Therefore, the Lagrangian function (6) is a convex function, and the optimization of the decision variable $t_{1}$ must be the corner-point solution that can be easily calculated as $\pi\left(t_{1}=t_{0}\right)=\delta q F(T)>\pi\left(t_{1}=T\right)=0$. Therefore, the 
optimization of $t_{1}$, which is denoted as $t_{1}^{*}$, is equal to $t_{0}$ (i.e., $\left.t_{1}^{*}=0\right)$.

$$
\begin{aligned}
& f^{\prime}\left(t_{1}\right)\left\{\lambda\left[\int_{t_{0}}^{T} f(t) d t-2 \int_{t_{0}}^{t_{1}} f(t) d t\right]-\delta q\right\} \\
& >2 \lambda f^{2}\left(t_{1}\right) .
\end{aligned}
$$

Proposition 1. The provider releases the new version as soon as possible if the consumers' perceived value of the mobile app is not sufficiently high.

Equation (2) indicates that the consumers obtain a relative lower utility when their perceived value of the mobile app is not sufficiently high, and a lower utility drives the monopolist to charge a lower price. This situation is disadvantageous for the profit of the monopolist. In this context, it is optimal for the monopolist to launch the new version as soon as possible to yield profits within a short time.

4.2. The Value of $\delta$ Is Sufficiently High. When the value of the parameter $\delta$ is sufficiently high, technically, $\delta>$ $(1 / q)\left\{\lambda\left[\int_{t_{0}}^{T} f(t) d t-2 \int_{t_{0}}^{t_{1}} f(t) d t\right]-2 \lambda f^{2}\left(t_{1}\right) / f^{\prime}\left(t_{1}\right)\right\}$, equation (12) is established, and we can derive that $\partial^{2} L / \partial t_{1}^{2}<0$. Therefore, the Lagrangian function (6) has the maximal value.

$$
\begin{aligned}
& f^{\prime}\left(t_{1}\right)\left\{\lambda\left[\int_{t_{0}}^{T} f(t) d t-2 \int_{t_{0}}^{t_{1}} f(t) d t\right]-\delta q\right\} \\
& <2 \lambda f^{2}\left(t_{1}\right) .
\end{aligned}
$$

However, the transcendental nature of $\partial L / \partial t_{1}$ precludes the derivation of a closed-form solution of $t_{1}^{*}$. Therefore, we conduct a sensitivity analysis of the parameter $\lambda$ using the implicit function theorem [23]. By letting $H\left(t_{1}^{*}, \lambda, \delta\right)=$ $\partial L / \partial t_{1}^{*}$, we can obtain the expression $H\left(t_{1}, \lambda, \delta\right)$ in (11). The first-order derivative of $t_{1}^{*}$ with respect to $\lambda$ is derived using (12).

$$
\begin{aligned}
& H\left(t_{1}^{*}, \lambda, \delta\right)=f\left(t_{1}^{*}\right)\left[\lambda F(T)-2 \lambda F\left(t_{1}^{*}\right)-\delta q\right]=0 \\
& \frac{\partial t_{1}^{*}}{\partial \lambda}=-\frac{\partial H / \partial \lambda}{\partial H / \partial t_{1}^{*}} \\
& \quad=-\frac{f\left(t_{1}^{*}\right)\left[F(T)-2 F\left(t_{1}^{*}\right)\right]}{f^{\prime}\left(t_{1}^{*}\right)\left[\lambda F(T)-2 \lambda F\left(t_{1}^{*}\right)-\delta q\right]-2 \lambda f^{2}\left(t_{1}^{*}\right)} .
\end{aligned}
$$

Given that $t_{1}^{*}$ satisfies $\partial L / \partial t_{1}^{*}=0, f\left(t_{1}^{*}\right)\left[\lambda F(T)-2 \lambda F\left(t_{1}^{*}\right)-\right.$ $\delta q]=0$. We obtain $f\left(t_{1}^{*}\right)>0$ and derive $\lambda F(T)-2 \lambda F\left(t_{1}^{*}\right)-$ $\delta q=0$. Consequently, $\partial t_{1}^{*} / \partial \lambda=\delta q / 2 \lambda^{2} f\left(t_{1}^{*}\right)$ and $\partial t_{1}^{*} / \partial \lambda>$ 0 .

Proposition 2. The optimal launch timing of a new version of mobile app increases with the intensity of network effect. That is, the vendor delays introducing the upgrading versions if the intensity of network effect is strong.
When the intensity of network effect becomes stronger, it is optimal for the app publisher to prolong the sales timing of the old version. A longer sales timing could improve the adoption rate of the old version. Consequently, stronger intensity of network effect, together with the larger user-base, makes the consumers enjoy more utility. From (3), we can induce that a higher utility level permits the monopolist to charge a higher price. Therefore, the monopolist should delay launching new product to extract more consumers' surplus.

Let us turn our analysis to parameter $\delta$. The first-order derivative of $t_{1}^{*}$ with respect to $\lambda$ is derived in (14). Given that $\lambda F(T)-2 \lambda F\left(t_{1}^{*}\right)-\delta q=0$, we obtain $\partial t_{1}^{*} / \partial \delta=-q / 2 \lambda f\left(t_{1}^{*}\right)<$ 0 .

$$
\begin{aligned}
\frac{\partial t_{1}^{*}}{\partial \delta} & =-\frac{\partial H / \partial \delta}{\partial H / \partial t_{1}^{*}} \\
& =\frac{q f\left(t_{1}^{*}\right)}{f^{\prime}\left(t_{1}^{*}\right)\left[\lambda F(T)-2 \lambda F\left(t_{1}^{*}\right)-\delta q\right]-2 \lambda f^{2}\left(t_{1}^{*}\right)} .
\end{aligned}
$$

Proposition 3. The optimal launching time of a new mobile app version decreases along with the consumers' perceived value. That is, the vendor accelerates the introducing of upgrading versions when the consumers enjoy a high perceived value.

A consumer with a higher perceived value can enjoy a better experience from the app. A better experience can also lead to a higher utility and a higher price that benefits the monopolist. In this condition, it is optimal for the vendor to launch new generations fast to generate profits within a relatively short time.

We then discuss the relationship between the optimal launch timing of $V_{1}\left(t_{1}^{*}\right)$ and the timing when $V_{0}$ is at maturity $(t)$. The first-order derivative of the noncumulative adoption rate $f(t)$ with respect to $t$ is presented in (15). The noncumulative adoption rate reaches its peak when $\widehat{t}=$ $-\ln (a / b) /(a+b)$.

$$
\begin{aligned}
& f^{\prime}(t) \\
& =\frac{m(a+b)^{3} e^{-(a+b) t}\left[(b / a) e^{-(a+b) t}+1\right]\left[(b / a) e^{-(a+b) t}-1\right]}{a\left[(b / a) e^{-(a+b) t}+1\right]^{4}} .
\end{aligned}
$$

The first-order derivative of the monopolist's profit $\pi$ with respect to $t$ is presented in

$$
\pi^{\prime}(t)=f(t)[\lambda F(T)-2 \lambda F(t)-\delta q]
$$

Given that $\pi^{\prime \prime}(t)<0$ and $\pi^{\prime}\left(t_{1}^{*}\right)=0$, we obtain that $\pi^{\prime}(\hat{t})>0$ if $\widehat{t}<t_{1}^{*}$ and $\pi^{\prime}(\hat{t})<0$ if $\widehat{t}>t_{1}^{*}$. We can easily calculate $\pi^{\prime}(\hat{t})=\left(m(a+b)^{2} / 4 b\right)[\lambda F(T)-m \lambda(1-a / b)-\delta q]$ and deduce that (I) $t_{1}^{*}<\widehat{t}$ when $\lambda<\delta q /(F(T)-m(1-a / b))$ and (II) $t_{1}^{*}>\widehat{t}$ when $\lambda>\delta q /(F(T)-m(1-a / b))$. These arguments are further expounded in the following propositions.

Proposition 4. The new version of the mobile app should be launched after (before) the old version reaches its maturity when the intensity of the network effect is (not) sufficiently strong. 


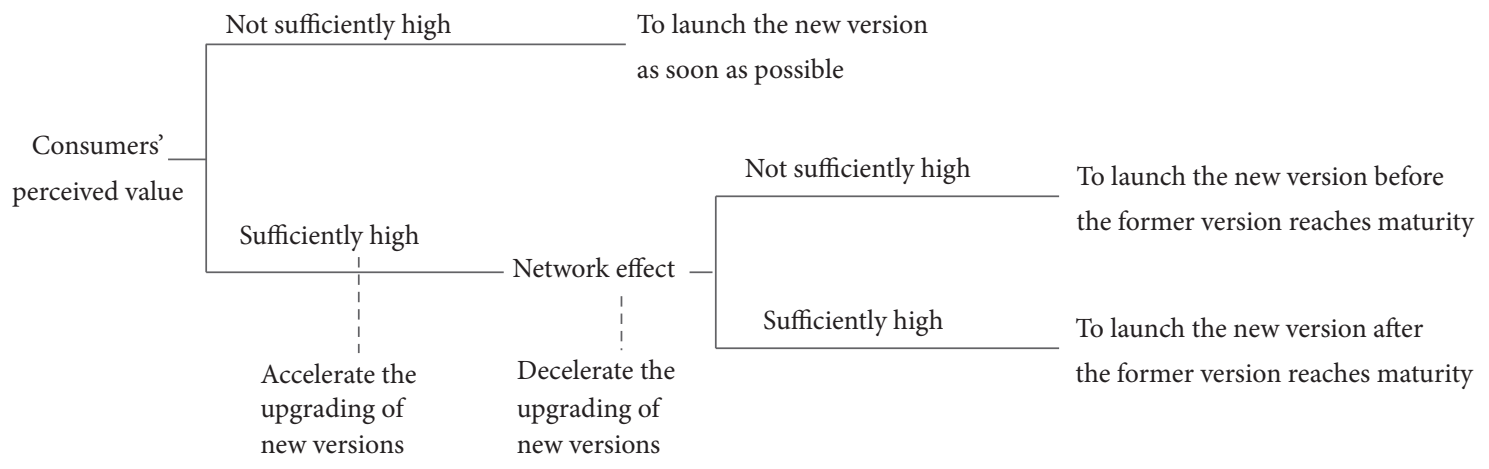

FIGURE 1: The summarization of the app vendor's decision of when to launch new versions.

If the intensity of the network effect is not sufficiently strong, then the consumers will obtain a relative low utility, which in turn leads to low prices that can harm the profits of the monopolist. Therefore, the vendor should accelerate its app upgrades and grab the consumer surplus as fast as possible. However, if the intensity of the network effect is very strong, then it is optimal for the vendor to delay launching its new product. The reason is that to delay launching new product expands the installed base of the old products and then allows the consumers to enjoy much more externality from other users. Hence, the more externality eventually allows the vendor to charge high prices for the new version and earn more profits.

\section{Conclusions}

5.1. Managerial Insights. The successive release of mobile apps is a complex task, and the timing of launching a new version is one of the most difficult decisions that a manager must make. We build an analytical model that is driven solely by economic considerations to study this problem. We conduct a sensitivity analysis of two significant parameters, namely, the intensity of the network effect and the coefficient of consumers' perceived value. The main implications of this analytical work are threefold. First, if the consumers' perceived value is not sufficiently high, an astute manager should adopt the "now" launching strategy (defined by [17]) and grab the consumers' surplus as soon as possible. Second, when the consumers perceive a sufficiently high value from the app, then the vendors' launching decision must take the network effect of products into account. Specifically, if the consumers enjoy a high network externality, then mobile app providers should adopt the strategy of "after maturity," which is launching the new version after the sales of its former generation reach maturity. Meanwhile, if the network effect of the app is not sufficiently strong, then the mobile app providers should adopt the strategy of "before maturity," which is to launch the new version before the sales of the former generation reach maturity. The strategy of "before maturity" and "after maturity" is defined by Joshi et al. [20]. Last but not least, the updating rate of new versions increases along with the consumers' perceived value and decreases along with the mobile app's network effect. The main results of this work are summarized in Figure 1.

5.2. Future Works. This work captures the impact of the network effect and the consumers' perceived value on the optimal entry timing of new app versions. Nevertheless, this work also has its limitations.

First, this study assumes that the market consists of homogeneous customers in terms of their evaluation of product features. A challenging extension is to jointly consider customer heterogeneity.

Second, this work discusses the marketing policy of app vendors from the perspective of consumers' value; hence, the revenue of vendors is limited to the fees from consumers. However, in the realistic market, many mobile app providers promise consumers with a free download and increase their profits from other avenues, such as ad revenues [24]. In our future work, we intend to extend our model by incorporating diverse profit avenues and explore the launching strategy more comprehensively.

Third, this work presents a theoretical formulation to analyze the market entry strategy of mobile app vendors but does not provide concrete examples for lacking of numerical values of parameters. However, the value of parameters could be estimated through the realistic operating data. For instance, Jiang et al. [19] estimate the value of the coefficient of innovation and imitation through the marketing data of analog and digital cellular phone in the US. In our future work, inspired by the prior literature, we intend to estimate the value of parameters from the marketing data provided by the app vendors who cooperate with us. Based on this, we would conduct an experience study to demonstrate the validity of this work.

Fourth, the proposed model can be further extended to examine a duopoly setting with two software providers. In a competitive market, firm decisions may be affected by new factors, such as consumer switching cost, as investigated by Mehra et al. [25].

Fifth, the findings from this study can be improved by incorporating development cost. Digital products often involve large up-front costs, which is an important economic consideration that the providers should consider. 


\section{Notations}

$\delta$ : Coefficient of consumers' perceived value

$\lambda$ : Intensity of network effect

$q$ : The inherent value of app

$t_{0}$ : Launch timing of the initial version

$t_{1}$ : Launch timing of the upgrade version

$m$ : Market potential

a: Coefficient of innovation

$b$ : Coefficient of imitation

$\pi$ : The vendor's cumulative profit

$T$ : The vendor's planning horizon.

\section{Conflicts of Interest}

The authors declare that they have no conflicts of interest.

\section{Acknowledgments}

The current paper is supported by Soft Science Research Program Project of Hebei Province (no. 16450124) and Research Foundation Program of Hebei University of Economics and Business (no. 2017KYY01).

\section{References}

[1] V. Krishnan and K. T. Ulrich, "Product development decisions: a review of the literature," Management Science, vol. 47, no. 1, pp. $1-21,2001$.

[2] B. Kahin and H. Varian, Versioning Information Goods, MIT Press, Cambridge, Mass, USA, 2000.

[3] R. B. Woodruff, "Customer value: the next source for competitive advantage," Journal of the Academy of Marketing Science, vol. 25, no. 2, pp. 139-153, 1997.

[4] H. K. Cheng and Y. Liu, "Optimal software free trial strategy: the impact of network externalities and consumer uncertainty," Information Systems Research, vol. 23, no. 2, pp. 488-504, 2012.

[5] X. Wei and B. R. Nault, "Experience information goods: "version-to-upgrade"," Decision Support Systems, vol. 56, no. 1, pp. 494-501, 2013.

[6] H. K. Cheng, S. Li, and Y. Liu, "Optimal software free trial strategy: limited version, time-locked, or hybrid?” Production Engineering Research and Development, vol. 24, no. 3, pp. 504517, 2015.

[7] H. K. Cheng and Q. C. Tang, "Free trial or no free trial: optimal software product design with network effects," European Journal of Operational Research, vol. 205, no. 2, pp. 437-447, 2010.

[8] F. Bass, "A new product growth model for consumer durables," Management Science, vol. 15, pp. 215-227, 1969.

[9] K. S. Moorthy and I. P. Png, "Market segmentation, cannibalization, and the timing of product introductions," Management Science, vol. 38, no. 3, pp. 345-359, 1992.

[10] A. Fishman and R. Rafael, "Product innovation by a durablegood monopoly," The RAND Journal of Economics, vol. 31, no. 2, pp. 237-252, 2000.

[11] K. Ramachandran and V. Krishnan, "Design architecture and introduction timing for rapidly improving industrial products," Manufacturing and Service Operations Management, vol. 10, no. 1, pp. 149-171, 2008.
[12] Q.-H. Wang and K.-L. Hui, "Delayed product introduction," Decision Support Systems, vol. 53, no. 4, pp. 870-880, 2012.

[13] A. Mehra, A. Seidmann, and P. Mojumder, "Product life-cycle management of packaged software," Production Engineering Research and Development, vol. 23, no. 3, pp. 366-378, 2014.

[14] V. Choudhary and Z. J. Zhang, "Research note-patching the cloud: the impact of saas on patching strategy and the timing of software release," Information Systems Research, vol. 26, no. 4, pp. 845-858, 2015.

[15] T. Ebina, N. Matsushima, and D. Shimizu, "Product differentiation and entry timing in a continuous time spatial competition model," European Journal of Operational Research, vol. 247, no. 3, Article ID 13056, pp. 904-913, 2015.

[16] A. Prasad, B. Bronnenberg, and V. Mahajan, "Product entry timing in dual distribution channels: the case of the movie industry," Review of Marketing Science, vol. 2, article no. 4, pp. $1-18,2004$.

[17] L. O. Wilson and J. A. Norton, "Optimal entry timing for a product line extension," Marketing Science, vol. 8, no. 1, pp. 117, 1989.

[18] V. Mahajan and E. Muller, "Timing, diffusion, and substitution of successive generations of technological innovations: the IBM mainframe case," Technological Forecasting \& Social Change, vol. 51, no. 2, pp. 109-132, 1996.

[19] Z. Jiang, S. Jiang, and D. C. Jain, "Now or never revisited: an analysis of market entry timing for successive product generation," in Proceedings of the 21st Americas Conference on Information Systems (AMCIS '15), Fajardo, Puerto Rico, August 2015.

[20] Y. V. Joshi, D. J. Reibstein, and Z. J. Zhang, "Optimal entry timing in markets with social influence," Management Science, vol. 55, no. 6, pp. 926-939, 2009.

[21] R. M. Krankel, I. Duenyas, and R. Kapuscinski, "Timing successive product introductions with demand diffusion and stochastic technology improvement," Manufacturing and Service Operations Management, vol. 8, no. 2, pp. 119-135, 2006.

[22] M. Xu and Y. Shang, "Kinship verification using facial images by robust similarity learning," Mathematical Problems in Engineering, vol. 2016, Article ID 4072323, 8 pages, 2016.

[23] A. de La Fuente, Mathematical Methods and Models for Economists, Cambridge University Press, Cambridge, UK, 2000.

[24] C.-L. Hsu and J. C.-C. Lin, "What drives purchase intention for paid mobile apps? - an expectation confirmation model with perceived value," Electronic Commerce Research and Applications, vol. 14, no. 1, pp. 46-57, 2015.

[25] A. Mehra, R. Bala, and R. Sankaranarayanan, "Competitive behavior-based price discrimination for software upgrades," Information Systems Research, vol. 23, no. 1, pp. 60-74, 2012. 


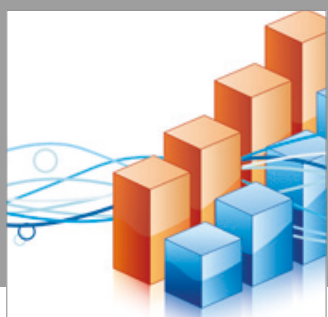

Advances in

Operations Research

vatersals

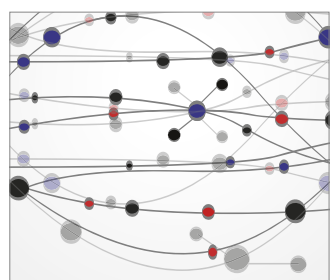

\section{The Scientific} World Journal
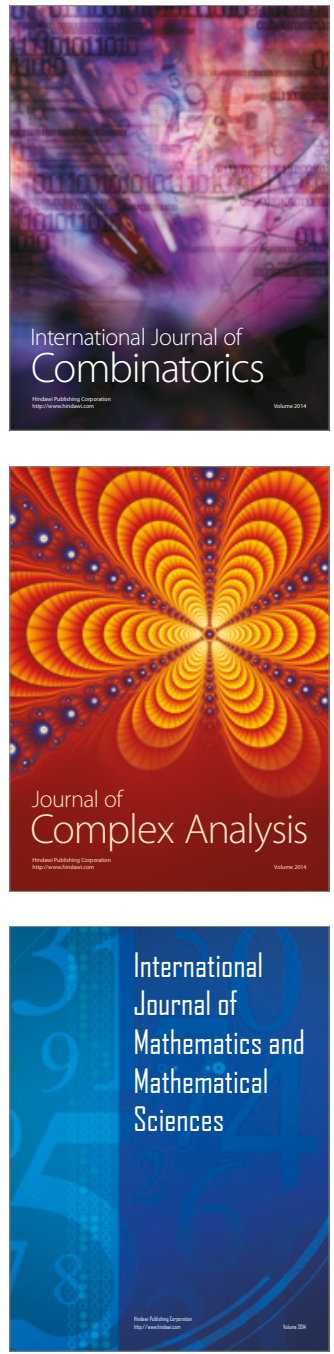
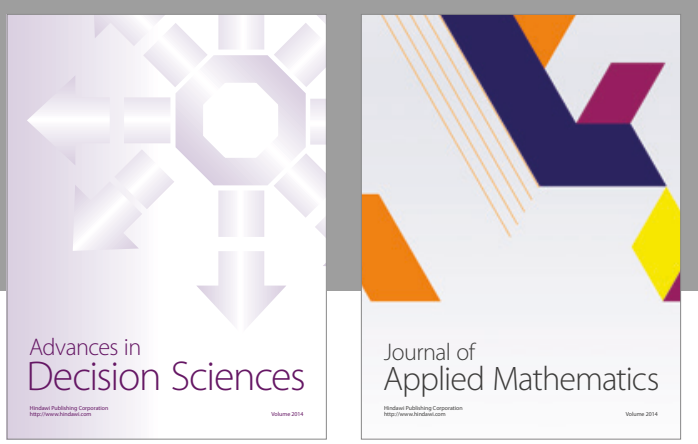

Algebra

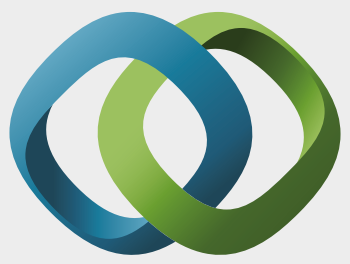

\section{Hindawi}

Submit your manuscripts at

https://www.hindawi.com
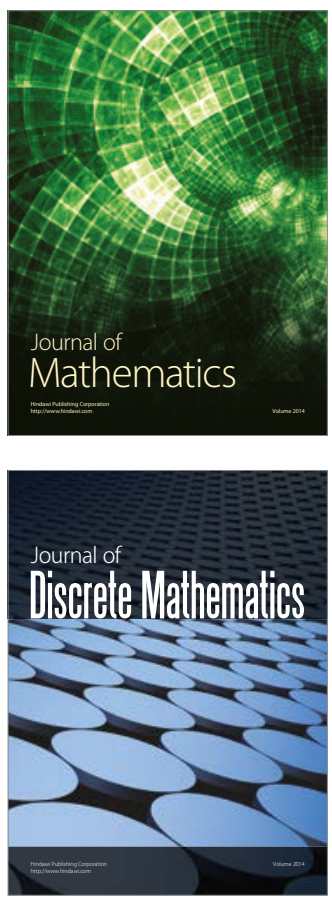

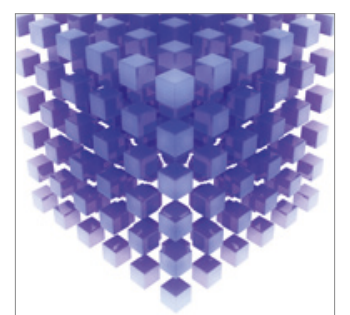

Mathematical Problems in Engineering
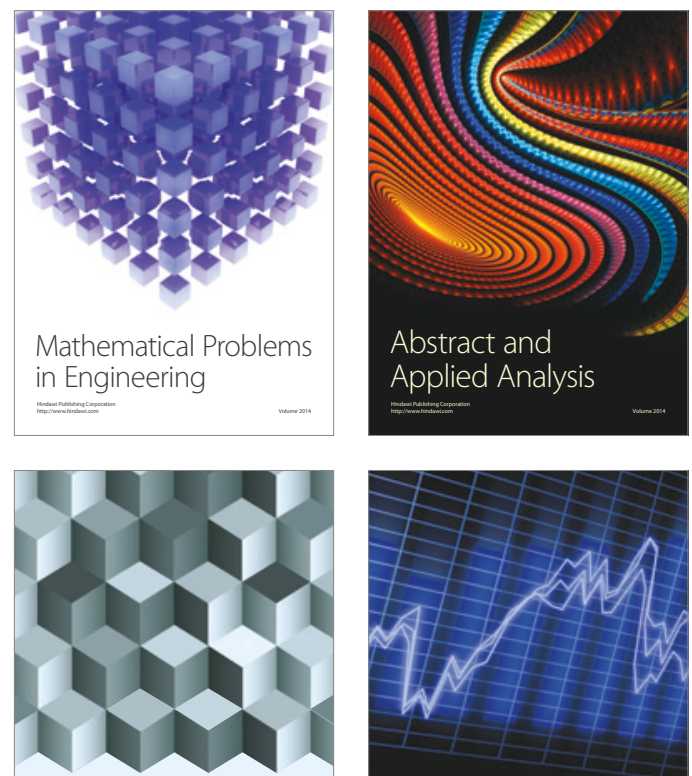

Journal of

Function Spaces

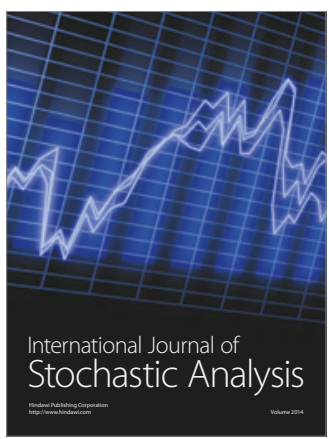

Probability and Statistics
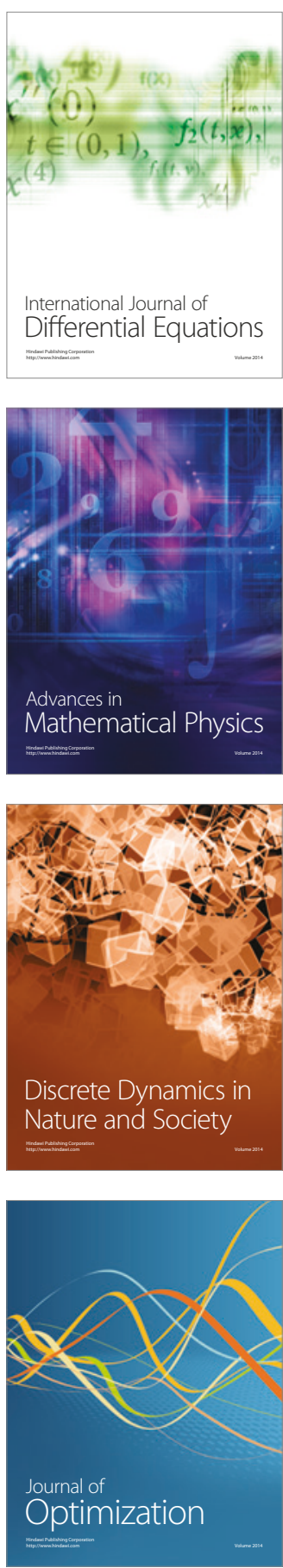